\title{
Stable Microtubule Subsets Facilitate Early HIV-1 Infection
}

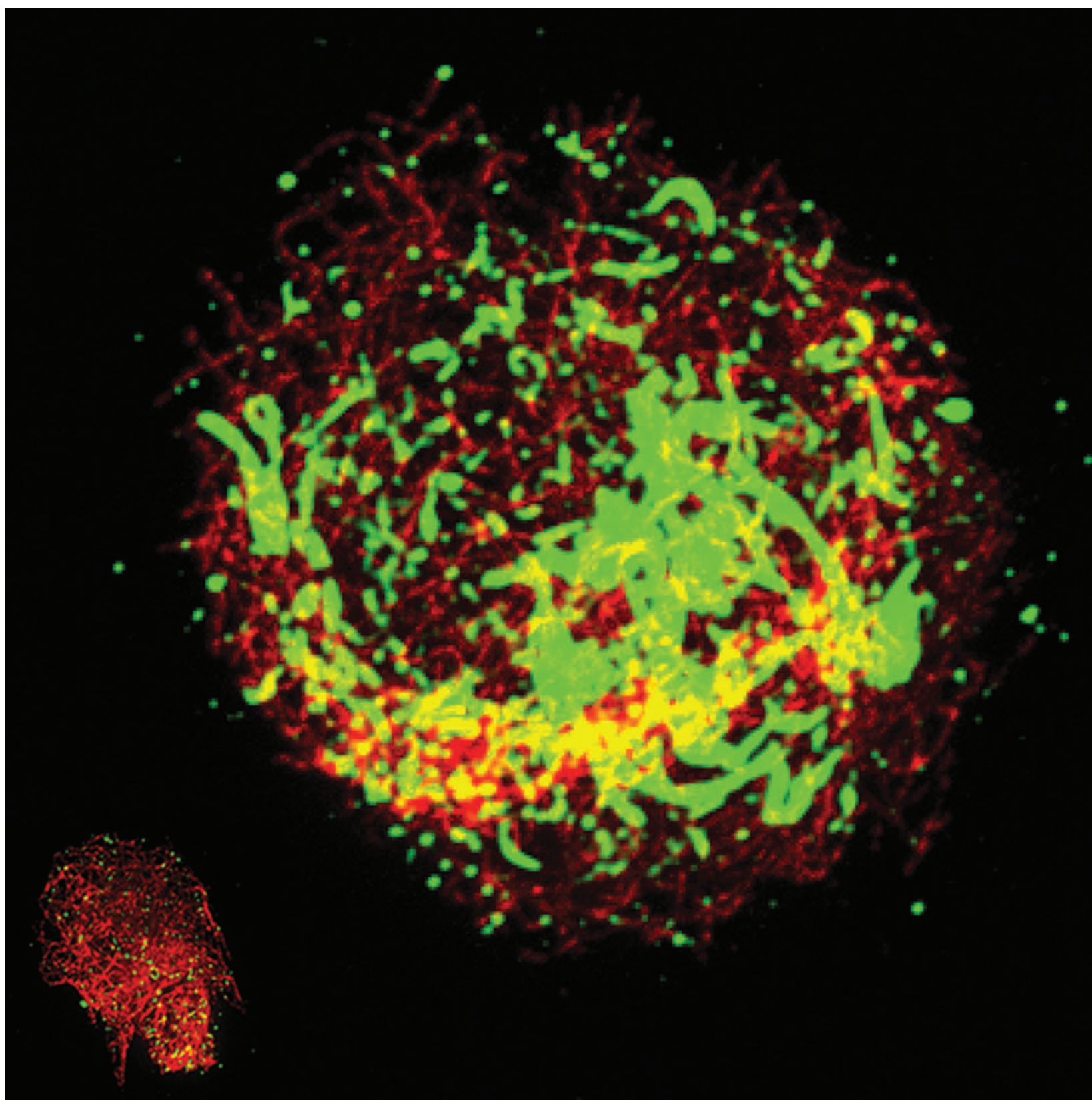

FIG. 1. HIV-1 induces stable microtubule (MT) formation in primary human macrophages. Primary human macrophages were mock infected (inset image lower left) or infected with pseudotyped HIV-1 and then fixed and stained for tyrosinated (red) and acetylated (green) tubulin, representative of dynamic and stable MTs, respectively. 
T HE DENSITY OF THE HOST CYTOPLASM presents pathogens such as HIV-1 with a major impediment to reaching the nucleus. As such, they must exploit host cytoskeletal networks to facilitate their movement. ${ }^{1}$ Within host cells, microtubule (MT) filaments act as "highways" for the intracellular transport of cargoes mediated by MT-associated motor proteins. ${ }^{2}$ While it has been established that the MT motor dynein mediates inward movement of HIV-1 to the nucleus, ${ }^{3}$ studies using MT depolymerizing agents such as nocodazole have produced seemingly conflicting suggestions that MTs are not important for HIV-1 infection. Recent findings relating to the role of specialized stable MT subsets now offer a potential explanation.

MTs form polarized filaments; their minus ends are anchored in a perinuclear MT organizing center (MTOC) while their plus ends grow outward to explore or "sense" the intracellular environment and form connections to the cell periphery. As such, in many cell types MTs are highly dynamic, undergoing rapid phases of growth and collapse through the addition and loss of tubulin subunits primarily at the plus end. MT stability is controlled by highly specialized plus-end-binding proteins, called + TIPs. Central to this is the end-binding protein EB1, which recognizes the MT plus end and recruits other+ TIPs that control MT dynamics. In response to specific signals, subsets of MTs become stabilized and form long-lived filaments that acquire distinguishing posttranslational tubulin modifications such as detyrosination and acetylation. Although these modifications do not seem to impart stability per se, they are thought to enable selective recognition of stable MT subsets by specific MT motor proteins. As such, these filaments can act as specialized tracks for vesicle trafficking to facilitate processes such as cell polarization. A notable feature of these long-lived MT subsets is their relative insensitivity to MT depolymerizing agents, but their potential contribution to HIV-1 infection has until now gone largely unstudied.

We recently found that HIV-1 infection rapidly induces MT stabilization in a range of human cell types, ${ }^{4}$ including natural target cells such as $\mathrm{T}$ cells, microglia, and primary macrophages (Fig. 1). This stabilization appears to occur very soon after viral entry and involves viral targeting of EB1 and the EB1-binding + TIP, Kif4. MT stabilization continues to increase as infection progresses, potentially as more virus enters the cell, triggered by viral proteins released during uncoating or by the activation of signal pathways that regulate MT stability, something we are currently studying. Viral particles also exhibited significant localization to stable MTs and when stable MT formation was blocked using either RNA interference (RNAi) or dominant negative approaches infection was potently suppressed, with viral particles failing to reach the nucleus efficiently. These findings suggest that HIV-1 induces the formation of specialized stable MT subsets early after entry in order to facilitate translocation of viral particles to the nucleus. The importance of these stable MT subsets to early HIV-1 infection offers an explanation for why dynein is important for infection yet MT depolymerizing agents have little effect, and identifies new factors to explore as potential therapeutic targets to treat HIV-1.

\section{Acknowledgment}

This work was supported by NIH Grant GM101975.

\section{References}

1. Naghavi MH and Goff SP: Retroviral proteins that interact with the host cell cytoskeleton. Curr Opin Immunol 2007; 19:402-407.

2. Dodding MP and Way M: Coupling viruses to dynein and kinesin-1. EMBO J 2011;30:3527-3539.

3. McDonald D, Vodicka MA, Lucero G, et al.: Visualization of the intracellular behavior of HIV in living cells. J Cell Biol 2002;159:441-452.

4. Sabo Y, Walsh D, Barry DS, et al.: HIV-1 induces the formation of stable microtubules to enhance early infection. Cell Host Microbe 2013;14:535-546.

Address correspondence to: Mojgan H. Naghavi Department of Biochemistry and Molecular Biophysics Columbia University New York, New York 10032

E-mail:mn2034@columbia.edu 